\title{
A New 3D 6-Subiteration Thinning Algorithm Based on $P$-Simple Points
}

\author{
Christophe Lohou and Gilles Bertrand \\ Laboratoire d'Algorithmique et Architecture des Systèmes Informatiques $\left(A^{2} \mathrm{SI}\right)$, \\ École Supérieure d'Ingénieurs en Électrotechnique et Électronique (EsIEE), \\ 2, bld Blaise Pascal, Cité Descartes, BP 99, F-93162 Noisy-le-Grand Cedex, France \\ \{lohouc, bertrang\}@esiee.fr
}

\begin{abstract}
In a recent study [1, we proposed a new methodology to build thinning algorithms based on the deletion of $P$-simple points. This methodology may permit to conceive a thinning algorithm $A^{\prime}$ from an existent thinning algorithm $A$, such that $A^{\prime}$ deletes at least all the points removed by $A$, while preserving the same end points.

In this paper, by applying this methodology, we propose a new 6 -subiteration thinning algorithm which deletes at least all the points removed by the 6-subiteration thinning algorithm proposed by Palágyi and Kuba [2].
\end{abstract}

\section{Introduction}

Some graphical applications require to transform objects while preserving their topology [23]. That leads to the well-known notion of simple point: a point in a binary image is said to be simple if its deletion from the image "preserves the topology" 4/5678,91011/2,1314. A process deleting simple points is called a thinning algorithm. During the thinning process, certain simple points are kept in order to preserve some geometrical properties of the object. Such points are called end points. The result obtained by a thinning algorithm is called a skeleton.

A process deleting simple points in parallel may not preserve the topology. For example, a two-width ribbon may vanish because all its points are simple. Therefore, a parallel thinning algorithm must use a "certain deletion strategy" in order to preserve the topology. For example, we may consider a deletion strategy based on subiterations, which consists in dividing a deletion iteration into several subiterations. These subiterations may be based on directions [15. 16,17218 , or on subgrids 1920]. Another example of deletion strategy consists in using an extended neighborhood; such a strategy may lead to fully parallel thinning algorithms [21,322].

One of the authors has proposed the notion of $P$-simple point [23]. A subset composed solely of $P$-simple points may be deleted in one time while preserving the topology. Furthermore, a $P$-simple point may be locally characterized, once $P$ is known. In a recent paper [1], we proposed a set $P^{x}$, locally defined for each point $x$ and from a set $P$. That has permitted us to propose a new

A. Braquelaire, J.-O. Lachaud, and A. Vialard (Eds.): DGCI 2002, LNCS 2301, pp. 102 2002. (C) Springer-Verlag Berlin Heidelberg 2002 


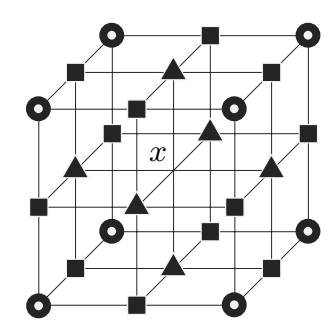

(a)

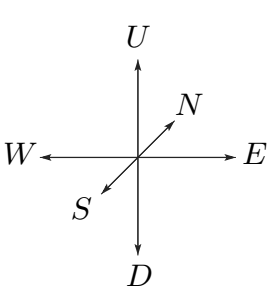

(b)

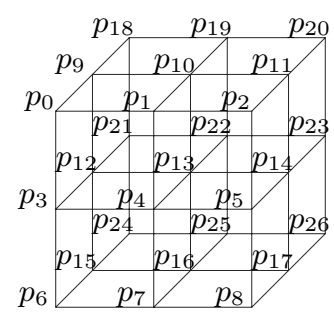

(c)

Fig. 1. (a) The 6-, 18-, and 26-neighbors of $x$, (b) the six major directions, (c) the used notations

thinning scheme, based on the deletion of $P^{x}$-simple points, which needs neither a preliminary step of labelling nor the examination of an extended neighborhood, in contrast to the already proposed thinning algorithms based on $P$-simple points [23].

In this paper, our purpose is to design a new 3D 6-subiteration thinning algorithm based on the deletion of $P^{x}$-simple points. We apply our general methodology proposed in [1]: from the 6-subiteration thinning algorithm devised by Palágyi and Kuba [2, we conceive a first thinning algorithm deleting $P^{x}$-simple points; then we improve it in such a way that it may delete at least all the points removed by the Palágyi and Kuba's thinning algorithm, while preserving the same end points.

\section{Basic Notions}

A point $x \in \mathbb{Z}^{3}$ is defined by $\left(x_{1}, x_{2}, x_{3}\right)$ with $x_{i} \in \mathbb{Z}$. We consider the three neighborhoods: $N_{26}(x)=\left\{x^{\prime} \in \mathbb{Z}^{3}: \operatorname{Max}\left[\left|x_{1}-x_{1}^{\prime}\right|,\left|x_{2}-x_{2}^{\prime}\right|,\left|x_{3}-x_{3}^{\prime}\right|\right] \leq 1\right\}$, $N_{6}(x)=\left\{x^{\prime} \in \mathbb{Z}^{3}:\left|x_{1}-x_{1}^{\prime}\right|+\left|x_{2}-x_{2}^{\prime}\right|+\left|x_{3}-x_{3}^{\prime}\right| \leq 1\right\}$, and $N_{18}(x)=\left\{x^{\prime} \in \mathbb{Z}^{3}:\right.$ $\left.\left|x_{1}-x_{1}^{\prime}\right|+\left|x_{2}-x_{2}^{\prime}\right|+\left|x_{3}-x_{3}^{\prime}\right| \leq 2\right\} \cap N_{26}(x)$. We define $N_{n}^{*}(x)=N_{n}(x) \backslash\{x\}$. We call respectively 6-, 18-, 26-neighbors of $x$ the points of $N_{6}^{*}(x), N_{18}^{*}(x) \backslash N_{6}^{*}(x)$, $N_{26}^{*}(x) \backslash N_{18}^{*}(x)$; these points are respectively represented in Fig. 11 (a) by black triangles, black squares, and black circles. The 6-neighbors of $x$ determine six major directions (Fig. 1 (b)): Up, Down, North, South, West, East; respectively denoted by $U, D, N, S, W$ and $E$. Each point of $N_{26}^{*}(x)$ may characterize one direction amongst the 26 that we can obtain from the 6 major ones; e.g. $S W$, $U S W \ldots$ Let Dir denote one of these 26 directions. The point in $N_{26}^{*}(x)$ along the direction Dir is called the Dir-neighbor of $x$ and is denoted by $\operatorname{Dir}(x)$. In the following, points in $N_{26}(x)$ are often denoted by $p_{i}$, with $0 \leq i \leq 26$ (Fig. 11 (c)); for example, $p_{0}$ is the $U S W$-neighbor of $p_{13}$, i.e. $p_{0}=\overline{U S W}\left(p_{13}\right)$. Let $X \subseteq \mathbb{Z}^{3}$. The points belonging to $X$ (resp. $\bar{X}$, the complement of $X$ in $\mathbb{Z}^{3}$ ) are called black points (resp. white points).

Two points $x$ and $y$ are said to be $n$-adjacent if $y \in N_{n}^{*}(x)(n=6,18,26)$. An $n$-path is a sequence of points $x_{0}, \ldots, x_{k}$, with $x_{i} n$-adjacent to $x_{i-1}$ and 


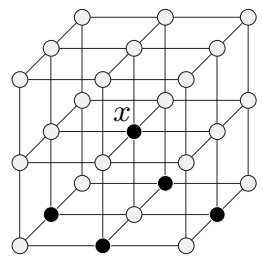

(a)

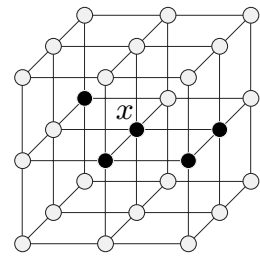

(b)

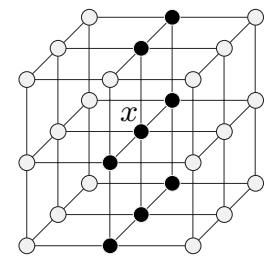

(c)

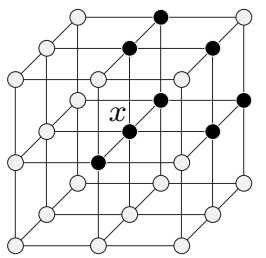

(d)

Fig. 2. Points belonging to $X$ and $\bar{X}$ are respectively represented by black discs and white circles. Only the point $x$ in (d) is 26 -simple

$1 \leq i \leq k$. If $x_{0}=x_{k}$, the path is closed. Let $X \subseteq \mathbb{Z}^{3}$. Two points $x \in X$ and $y \in X$ are $n$-connected if they are linked by an $n$-path included in $X$. The equivalence classes relative to this relation are the $n$-connected components of $X$. If $X$ is finite, the infinite connected component of $\bar{X}$ is the background, the other connected components of $\bar{X}$ are the cavities. In order to have a correspondence between the topology of $X$ and the one of $\bar{X}$, we have to consider two differents kinds of adjacency for $X$ and for $\bar{X}$ [6]: if we use an $n$-adjacency for $X$, we have to use another $\bar{n}$-adjacency for $\bar{X}$. In this paper, we only consider $(n, \bar{n})=(26,6)$. The presence of an $n$-hole in $X$ is detected whenever there is a closed $n$-path in $X$ that cannot be deformed, in $X$, into a single point (see [5], for further details). For example, a hollow ball has one cavity and no hole, a solid torus has one hole and no cavity, and a hollow torus has one cavity and two holes.

Let $X \subseteq \mathbb{Z}^{3}$. A point $x \in X$ is said to be $n$-simple if its removal does not "change the topology" of the image, in the sense that there is a one to one correspondence between the components, the holes of $X$ and $\bar{X}$ and the components, the holes of $X \backslash\{x\}$ and $\bar{X} \cup\{x\}$ (see [5], for a precise definition). The set composed of all $n$-connected components of $X$ is denoted by $\mathcal{C}_{n}(X)$. The set of all $n$-connected components of $X$ and $n$-adjacent to a point $x$ is denoted by $\mathcal{C}_{n}^{x}(X)$. Let $\# X$ denote the number of elements which belong to $X$. The topological numbers relative to $X$ and $x$ are the two numbers [10]: $T_{6}(x, X)=$ $\# \mathcal{C}_{6}^{x}\left[N_{18}^{*}(x) \cap X\right]$ and $T_{26}(x, X)=\# \mathcal{C}_{26}\left[N_{26}^{*}(x) \cap X\right]$. These numbers lead to a very concise characterization of 3D simple points [24]: $x \in X$ is 26 -simple for $X$ if and only if $T_{26}(x, X)=1$ and $T_{6}(x, \bar{X})=1$. Some examples are given in Fig. 2. The topological numbers relative to $x$ and $X$ or $\bar{X}$ are: $\left(T_{26}(x, X), T_{6}(x, \bar{X})\right)=$ $(1,2),(2,1),(1,2),(1,1)$ for the configurations (a), (b), (c) and (d), respectively. Only the configuration in Fig. 2 (d) corresponds to a 26-simple point.

\section{$3 \quad P$-Simple Points}

Let us introduce the notions of $P$-simple point and $P$-simple set 23 . In the following, we consider a subset $X$ of $\mathbb{Z}^{3}$, a subset $P$ of $X$, and a point $x$ of $P$. The point $x$ is $P$-simple (for $X$ ) if for each subset $S$ of $P \backslash\{x\}, x$ is 26simple for $X \backslash S$. Let $S(P)$ denote the set of all $P$-simple points. A subset $D$ 


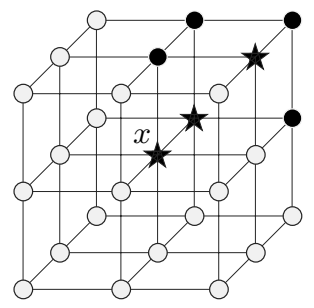

(a)

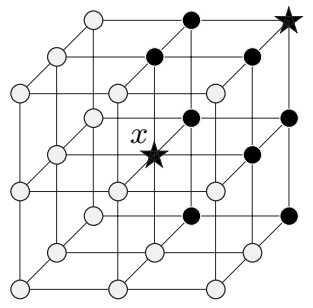

(b)

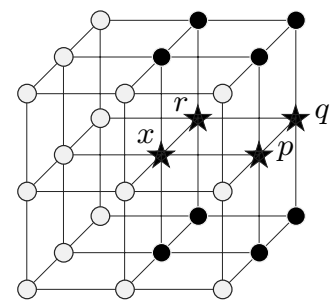

(c)

Fig. 3. Points belonging to $R, P$ and $\bar{X}$ are respectively represented by black discs, black stars and white circles. Only the points $x$ in (a) and (b) are $P$-simple

of $X$ is $P$-simple if $D \subseteq S(P)$. We have the remarkable property that any algorithm removing only $P$-simple subsets (i.e. subsets composed solely of $P$ simple points) is guaranteed to keep the topology unchanged 23 . We give a local characterization of a $P$-simple point [25] (see also [26]):

Proposition 1. Let $R$ denote the set $X \backslash P$. The point $x$ is P-simple iff: $\left\{\begin{array}{l}T_{26}(x, R)=1 \\ T_{6}(x, \bar{X})=1 \\ \forall(⿻,\end{array}\right.$

$\forall \forall \in N_{26}^{*}(x) \cap P, \exists z \in R$ such that $z$ is 26-adjacent to $x$ and to $y$,

$\forall y \in N_{6}^{*}(x) \cap P, \exists z \in \bar{X}$ and $\exists t \in \bar{X}$ such that $\{x, y, z, t\}$ is a unit square.

Some examples are given in Fig. 3; only the points $x$ in (a) and (b) are $P$-simple. Let us consider the subset $X$ depicted in Fig. 3 (c). The subset $S=\{p, q, r\}$ is a subset of $P \backslash\{x\}$; and $x$ is non-simple for $X \backslash S$. Therefore, by definition, the point $x$ cannot be a $P$-simple point; or directly with the Proposition 1 , the first $P$-simplicity condition is not verified because $T_{26}(x, R)=2$.

For each $x$ of $Z^{3}$, we consider a finite family $\mathcal{T}$ of pairs of subsets of $Z^{3}$ $\left(B^{k}(x), W^{k}(x)\right)$ with $1 \leq k \leq l$, such that $B^{k}(x) \cap W^{k}(x)=\emptyset$ and $x$ belongs to $B^{k}(x) ; \mathcal{T}$ is said to be a family of templates. In the following, we consider a subset $X$ of $\mathbb{Z}^{3}$. Let $P(\mathcal{T}, X)=\left\{x \in \mathbb{Z}^{3}: \exists k\right.$ with $1 \leq k \leq l$ such that $B^{k}(x) \subseteq$ $X$ and $\left.W^{k}(x) \subseteq \bar{X}\right\}$. In fact, $P(\mathcal{T}, X)$ corresponds to a Hit or Miss transform of $X$ by $\mathcal{T}$ [27 28].

A thinning algorithm, based on the deletion of $P$-simple points, usually considers subsets $P$ which may be characterized by a certain family $\mathcal{T}$ of templates. Such an algorithm must decide whether a point $x$ is $P(\mathcal{T}, X)$-simple or not: it must check if the point $x$ belongs to $P(\mathcal{T}, X)$, and in order to check the four conditions of the Proposition 1 it must check if the points $y$ of $N_{26}^{*}(x)$ belong to $P(\mathcal{T}, X)$. Such an algorithm may operate according to different ways to detect the points belonging to $P(\mathcal{T}, X)$ and the points being $P(\mathcal{T}, X)$-simple: it use either a preliminary step of labelling or the examination of an extended neighborhood [23] (see details in [1]). Note that a general strategy has already been proposed to design different thinning schemes and algorithms based on $P$-simple points [29] (see also [3031]). 
Let us introduce a subset $P^{x}$, locally defined for each point $x$ of $Z^{3}$ and from a set $P$ (described as previously by a family $\mathcal{T}$ of templates) [132]. From this subset, we will derive the notion of a $P^{x}$-simple point. For each $x$ of $\mathbb{Z}^{3}$, we define a new subset $P^{x}(\mathcal{T}, X)$ of $\mathbb{Z}^{3}$, determined by $P^{x}(\mathcal{T}, X)=\left\{y \in N_{26}(x): \exists k\right.$ with $1 \leq k \leq l$ such that $\left[B^{k}(y) \cap N_{26}(x)\right] \subseteq X$ and $\left.\left[W^{k}(y) \cap N_{26}(x)\right] \subseteq \bar{X}\right\}$. In fact, $P^{x}(\mathcal{T}, X)$ is constituted by the points $y$ of $N_{26}(x) \cap X$ which "may belong" to $P(\mathcal{T}, X)$, by the only inspection of membership to $X$ or to $\bar{X}$ of points belonging to $\left[B^{k}(y) \cup W^{k}(y)\right] \cap N_{26}(x)$. We have $P^{x}(\mathcal{T}, X) \supseteq\left[P(\mathcal{T}, X) \cap N_{26}(x)\right]$.

We have proven that a $P^{x}(\mathcal{T}, X)$-simple point is $P(\mathcal{T}, X)$-simple [32]. This implies that an algorithm deleting in parallel $P^{x}(\mathcal{T}, X)$-simple points is guaranteed to preserve the topology, because it deletes $P(\mathcal{T}, X)$-simple subsets. In addition, since $P^{x}(\mathcal{T}, X)$ is completely known in $N_{26}(x)$ for each point $x$, that permits us to propose a new thinning scheme, based on the deletion of $P^{x}(\mathcal{T}, X)$ simple points $x$, which needs neither a preliminary step of labelling nor the examination of an extended neighborhood, in contrast to the already proposed thinning algorithms based on $P(\mathcal{T}, X)$-simple points (see 1 for further details). In Sect. [5] we will propose a thinning algorithm deleting $P^{x}(\mathcal{T}, X)$-simple points.

Notations: In the following, we write $P$ (resp. $\left.P^{x}\right)$ instead of $P(\mathcal{T}, X)$ (resp. $\left.P^{x}(\mathcal{T}, X)\right)$ and " $x$ is a $P$-simple point (resp. $P^{x}$-simple point) means " $x$ is a $P(\mathcal{T}, X)$-simple point (resp. $P^{x}(\mathcal{T}, X)$-simple point)".

\section{Description of the Used Thinning Algorithms}

A thinning scheme consists in the repetition until stability of deletion iterations. In the case of 6-subiteration thinning algorithms, an iteration is divided into 6 subiterations, each of them successively corresponding to one of the 6 following directions: Up, Down, North, South, East and West (see Fig. 11 (b)). Let $\alpha$ denotes such a direction. The stability is obtained when there is no more deletion during 6 successive subiterations. Such a thinning scheme can be described by $X^{i}=X^{i-1} \backslash \operatorname{DEL}\left(X^{i-1}, \alpha\right)$ for the $i$ th deletion subiteration $(i>0)$, with $X^{0}=X$, and $\operatorname{DEL}(Y, \alpha)$ being the set of points to be deleted from $Y$, according to the direction $\alpha$ corresponding to the $i$ th subiteration. The stability is obtained when $X^{k}=X^{k+6}$.

Palágyi and Kuba have proposed a 6 -subiteration thinning algorithm [2], denoted by $\mathrm{PK}$ in the following. A set of $3 \times 3 \times 3$ matching templates is given for each direction. For a given direction $\alpha$, a point is deletable by PK if at least one template (or theirs rotations around the axis along the direction $\alpha$ ) in the set of templates matches it. The set of templates used by PK along the direction $\alpha$, is denoted by $\mathcal{T}_{\alpha}$ and is represented in Fig. 5 for the direction $\alpha=U$; see notations in Fig. 4. The templates for the other directions can be obtained by appropriate rotations and reflections of these templates. Sometimes, we will write that " $\mathcal{T}_{\alpha}$ deletes a point" to mean PK deletes this point during a subiteration along the direction $\alpha$. We recall the definition of an end point, adopted in [2], that we will also use in our proposed algorithm. A black point $x$ is an end point if the set $N_{26}^{*}(x)$ contains exactly one black point. We note that end points are prevented 
A position marked by a matches a black point. A position marked by a $\bigcirc$ matches a white point. At least one position marked by a $\boldsymbol{\nabla}$ belongs to $X$. Every position non marked matches either a black or a white point. A position marked by a $\star$ matches a black point belonging to a considered set $P$.

Fig. 4. Notations used in the following of the paper
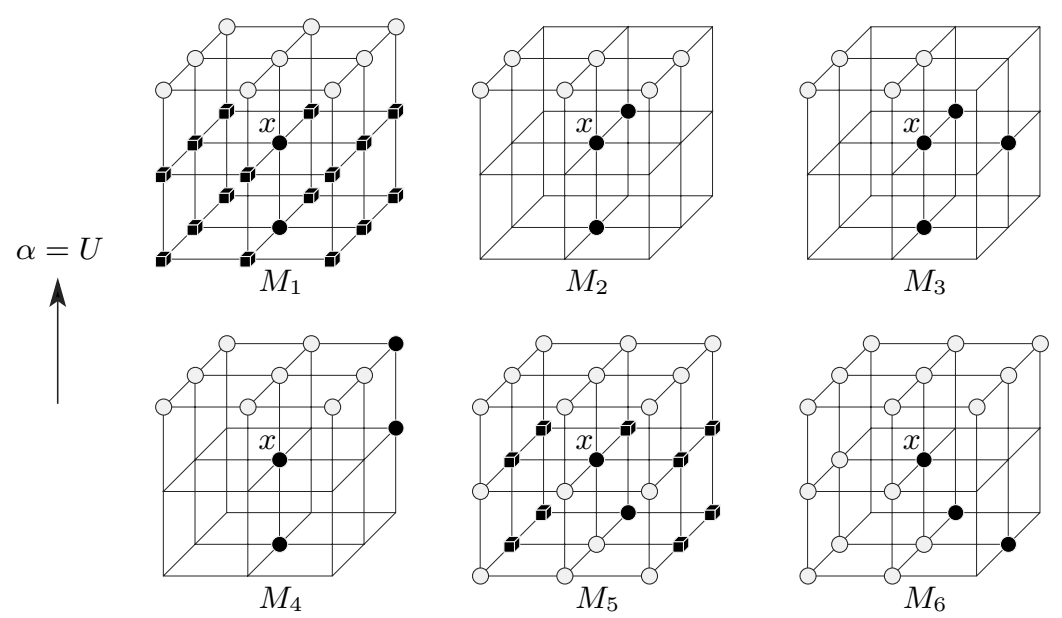

Fig. 5. The set $\mathcal{T}_{U}$ of thinning templates for the direction $U$, up to rotations around the vertical axis (see notations in Fig. 4)

to be deleted by the templates of $\mathcal{T}_{\alpha}$. According to the previous general thinning scheme (described in the beginning of this section), $D E L(Y, \alpha)$ is the set of points of $Y$ such that at least one of the templates of $\mathcal{T}_{\alpha}$ matches them, for the direction $\alpha$ corresponding to the deletion subiteration.

A 6 -subiteration thinning algorithm removing $P$-simple points, has already been proposed [23]. Now, we give a general scheme for 6 -subiteration thinning algorithms deleting $P^{x}$-simple points. It can be described by the scheme of the beginning of this section with $D E L(Y, \alpha)=S\left(P^{x}\right) ; S\left(P^{x}\right)$ being the set of $P^{x}$ simple points for $Y$ which are not end points and according to the direction $\alpha$ corresponding to the deletion subiteration. From this scheme, we will propose our algorithm by defining an appropriate $P$ (Sect. (5)), in the sense that we investigate $P$ such that our algorithm deletes at least all the points removed by PK. In the following, we write LB to indicate our final algorithm which deletes $P^{x}$-simple points, while preserving end points.

See [1] for details concerning the efficient implementation of such algorithms, with the use of Binary Decision Diagrams [33 34,35]; in fact, PK and LB have the same computational complexity. 


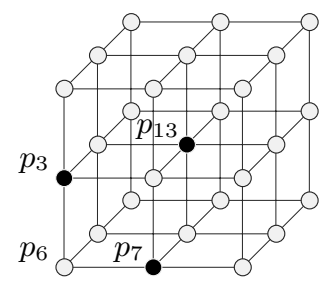

(a)

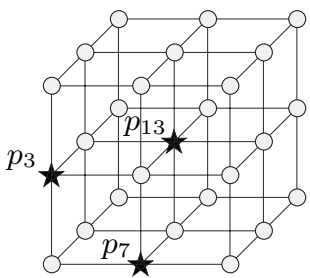

(b)

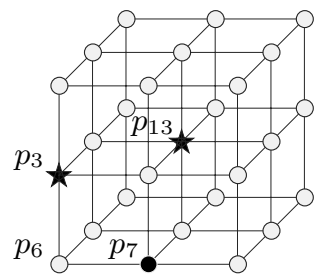

(c)

Fig. 6. This configuration (a) is not $P_{1}^{x}$-simple (b), and is $P_{2}^{x}$-simple (c)

\section{Our Thinning Algorithm (LB)}

In this section, we give the entire reasoning which leads us to propose two successive conditions of membership to a set $P$. The used methodology consists in proposing successive "refinements" of $P$, until to obtain a set $P$ such that at least all points deleted by PK are $P$-simple. This is achieved with our second proposal of a set $P$. We note that the first proposal, detailed in Sect. [5.1 is directly deduced from PK.

We first deal with the direction $U$ until a general comparison of our results. In the following, when we write "a point belongs to $P^{x}$ " then $x$ is the point $p_{13}$ for the considered configuration (see Fig. 1(c)). We write "a configuration is $P^{x}$-simple" to mean that the central point $x\left(=p_{13}\right)$ of this configuration is $P^{x}$-simple. Let $y$ be a point of a configuration, $y$ belongs to $\left\{p_{0}, \ldots, p_{26}\right\}$, see Fig. 1(c); we write "a point $y$ verifies a template $T$ " to mean that the template $T$ matches the configuration whose central point is $y$.

\subsection{First Membership Condition}

We observe that any point of $X$ deleted by $\mathcal{T}_{U}$ is such that its $U$-neighbor belongs to $\bar{X}$ (see templates in Fig. [5). Thus, we propose to consider $P_{1}=\{x \in X$ : the $U$-neighbor of $x$ belongs to $\bar{X}\}$. Among all $2^{26}$ possible configurations, we obtain 4423259 ones corresponding to $P_{1}^{x}$-simple and non end points, for the direction $U$.

Let us consider the configuration in Fig. 6 (a). The three points $p_{3}, p_{7}$ and $p_{13}$ belong to $P_{1}^{x}$ (Fig. [6 (b)) because they belong to $X$, and each $U$-neighbor of these points belongs to $\bar{X}$. The first and the third $P_{1}^{x}$-simplicity conditions are not verified for the central point $p_{13}$. Thus, the point $p_{13}$ is not $P_{1}^{x}$-simple. Nevertheless, it is matched by a rotation around the vertical axis of $M_{5}$ of $\mathcal{T}_{U}$. Therefore, it should be deleted by our wanted algorithm.

Let us examine the behavior of the other points of this configuration with the templates $\mathcal{T}_{U}$ (see Fig. [6 (a)). The point $p_{3}$ may verify a rotation around the vertical axis of $M_{5}$ or of $M_{6}$. The point $p_{7}$ cannot be deleted, because $p_{6}(=$ $\left.W\left(p_{7}\right)\right)$ belongs to $\bar{X}$ and $p_{3}\left(=U\left(p_{6}\right)\right)$ belongs to $X$, and the templates are such 


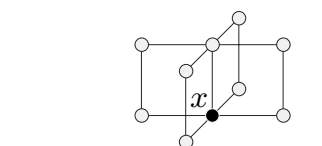

(a)

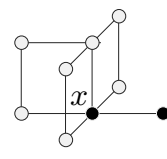

(b)

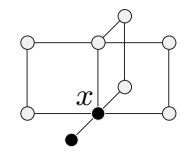

(c)

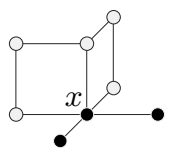

(d)

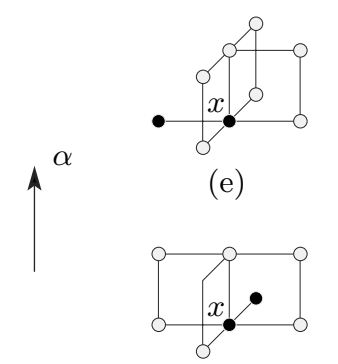

(e)

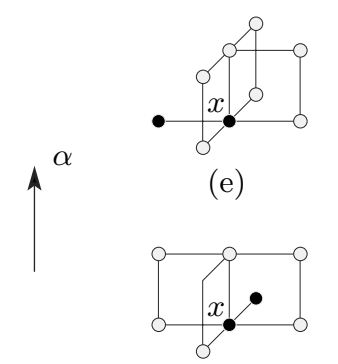

(i)

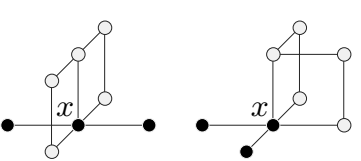

(f)

(g)
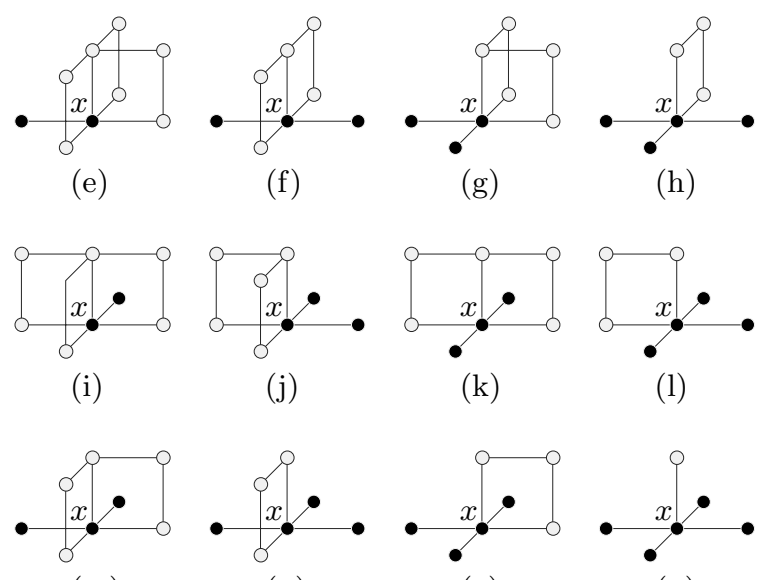

(m)

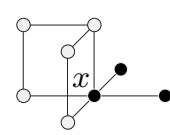

(j)

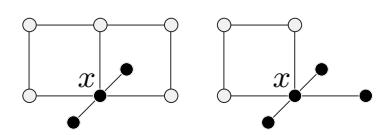

(k)
(1)

(p)

Fig. 7. A point $x$ belongs to $P_{2}$ iff it verifies at least one of these templates, according to the direction $\alpha$

that for any point $x$ deleted by $\mathcal{T}_{U}$ and for any $y$ belonging to $N_{6}^{*}(x) \cap \bar{X}, y$ being neither $U(x)$ nor $D(x)$, then the point $U(y)$ must belong to $\bar{X}$.

With these remarks, we can propose a new set $P_{2}$.

\subsection{Second Membership Condition}

We first introduce some notations. We recall that $\alpha$ denote one of the six deletion directions. Let $\bar{\alpha}$ denote the opposite direction. Let $N_{\alpha}^{6}(x)$ denote the four 6 neighbors of $x$ which belong to the $3 \times 3$ window perpendicular to the direction $\alpha$ and containing $x$ (in fact, $\left.N_{\alpha}^{6}(x)=N_{6}^{*}(x) \backslash\{\alpha(x), \bar{\alpha}(x)\}\right)$. We propose to consider $P_{2}=\{x \in X$ : the $\alpha$-neighbor of $x$ belongs to $\bar{X}$ and for any point $y$ belonging to $N_{\alpha}^{6}(x)$, if $y$ belongs to $\bar{X}$ then $\alpha(y)$ must belong to $\bar{X}$ \}, according to the direction $\alpha$.

With notations used in Sect. [3] the set $P_{2}$ can be described by the family composed of 16 pairs of subsets of $\mathbb{Z}^{3}\left(B^{k}(x), W^{k}(x)\right)$ with $1 \leq k \leq 16$, depicted in Fig. 7 for the direction $\alpha=U$; in fact, there are 6 main templates, up to rotations around the axis $(\alpha(x), \bar{\alpha}(x))$.

Let us consider the non $P_{1}^{x}$-simple configuration in Fig. 6 (b) (see notations in Fig. 6 (c)). The point $p_{13}$ belongs to $P_{2}^{x}$, as it verifies the template in Fig. 7 (a). The point $p_{3}$ belongs to $P_{2}^{x}$, as $p_{3}$ may verify the templates in Fig. 7 (a), (c), (e), or (g). The point $p_{7}$ does not belong to $P_{2}^{x}$ because there exists a 


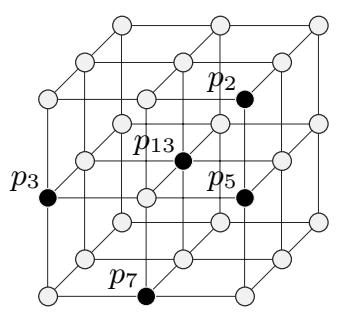

(a)

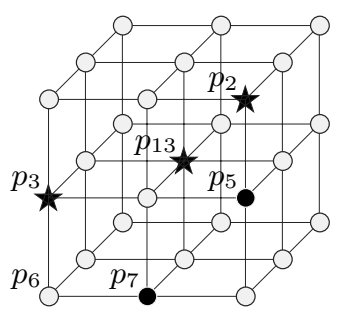

(b)

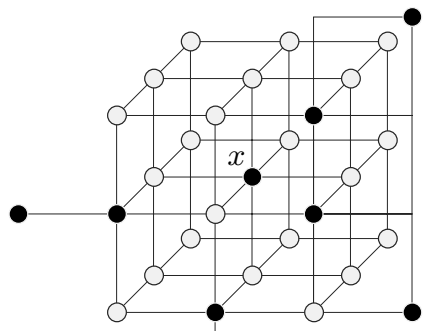

(c)

Fig. 8. (a) This configuration cannot be deleted by PK whatever the deletion direction, and is $P_{2}^{x}$-simple (b), in (c) (obtained from (a)) no point is deleted by PK nevertheless $x$ is deleted by LB

point $y\left(=p_{6}\left(=W\left(p_{7}\right)\right)\right)$ in $N_{U}^{6}\left(p_{7}\right) \cap N_{26}(x)$ which belongs to $\bar{X}$, and such that $p_{3}(=U(y))$ belongs to $X$; or more directly because $p_{7}$ verifies no template in Fig. 7. So, this non $P_{1}^{x}$-simple configuration (Fig. 6] (b)) is now $P_{2}^{x}$-simple (Fig. [6] (c)).

We obtain 6129527 configurations corresponding to $P_{2}^{x}$-simple and non end points, for the direction $U$. The 2124283 configurations deleted by $\mathcal{T}_{U}$, are also $P_{2}^{x}$-simple. The fact that the configurations deletable by PK are $P_{2}^{x}$-simple (for each direction and therefore for the whole algorithm) guarantees that the topology is preserved by PK (as PK deletes subsets of $P_{2}^{x}$-simple points, see Sect. 3 ).

For a better comparison between PK and LB, we generate the configurations deleted by these algorithms for each direction: PK deletes 9916926 configurations, i.e. there exists at least one deletion direction such that a given configuration among these ones is deleted for this direction by PK; LB deletes 23721982 configurations (139.2\% "better"). We recall that there are 25985118 simple and non end points amongst the $67108864\left(=2^{26}\right)$ possible $3 \times 3 \times 3$ configurations.

The configuration depicted in Fig. 8 (a) cannot be deleted by PK, whatever the deletion direction. This configuration is $P_{2}^{x}$-simple (Fig. 8 (b)), with $\alpha=U$. Indeed, the point $p_{2}$ belongs to $P_{2}^{x}$ as $p_{2}$ may verify the templates in Fig. 7 (a), (b), (c) or (d); $p_{3}$ belongs to $P_{2}^{x}$ as $p_{3}$ may verify the templates in Fig. 7 (a), (c), (e) or (g); $p_{13}$ belongs to $P_{2}^{x}$ as it verifies the template in Fig. 7 (a); $p_{5}$ does not belong to $P_{2}^{x}$ as $p_{2}\left(=U\left(p_{5}\right)\right)$ belongs to $X$; and $p_{7}$ does not belong to $P_{2}^{x}$ as there exists a point $y\left(=p_{6}\left(=W\left(p_{7}\right)\right)\right)$ in $N_{U}^{6}\left(p_{7}\right) \cap N_{26}(x)$ which belongs to $\bar{X}$ and such that $U(y)\left(=p_{3}\right)$ belongs to $X$ (or more directly, as $p_{7}$ verifies no template in Fig. (7).

The figure 8 (c) shows an image built from the configuration in Fig. 8] (a) such that each point is either a non simple point (except $x$ ) or an end point, and no point can be deleted by PK, nevertheless the point $x$ is deleted by LB. 


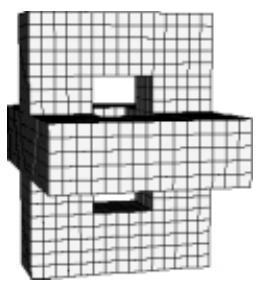

Initial object

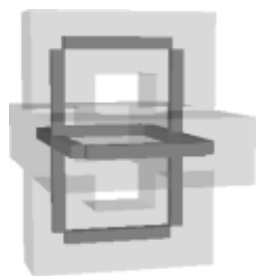

PK: $16-2256$

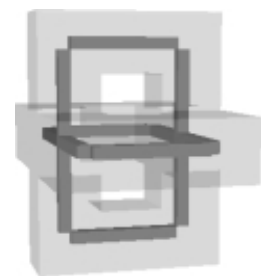

LB: $16-2256$

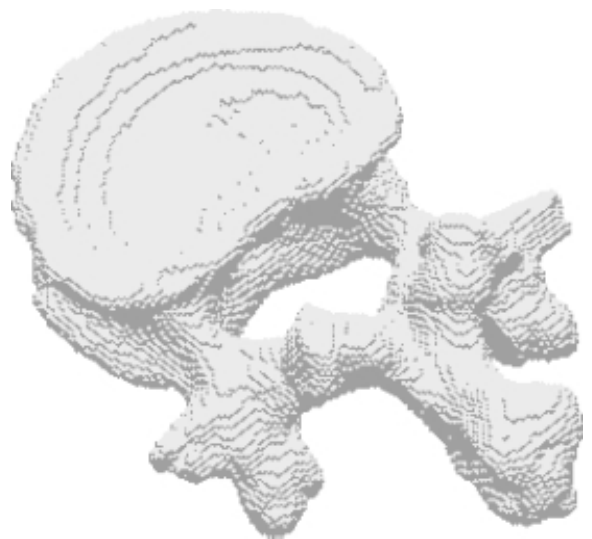

Initial object

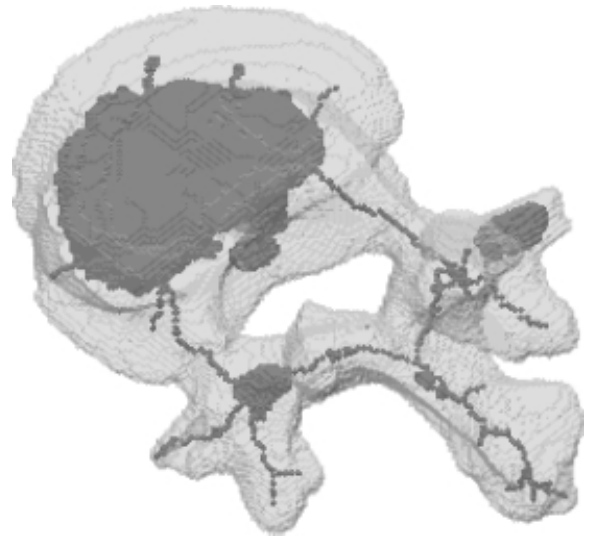

PK: $142-170001$

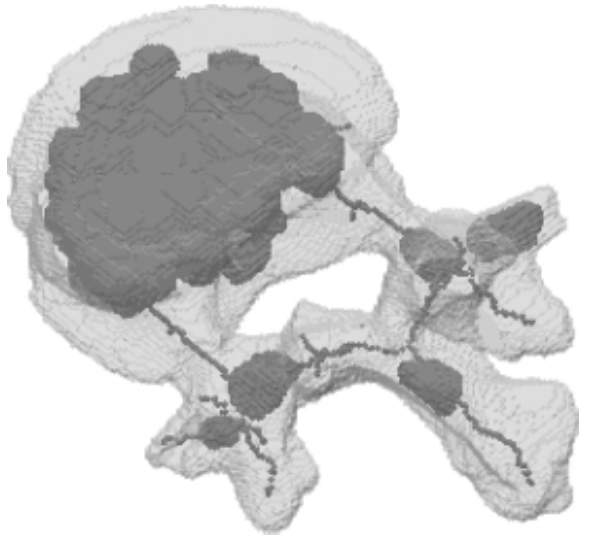

LB: $73-163874$

Fig. 9. Skeletons of a synthetic object and of a vertebra, with PK and LB. Under each figure are given the number of the last subiteration of deletion and the number of deleted points

\subsection{Results}

The skeletons of some images, obtained respectively by PK and LB are shown in Fig. 9. We observe that the number of deletion subiterations required by LB is less than or equal to the one of PK. The number of points deleted by LB is less 
than or equal to the one of PK. We recall that it is possible that LB needs more subiterations to obtain a skeleton than PK needs (see Fig. 8 (c)).

\section{Conclusion}

We have conceived a new 6 -subiteration thinning algorithm, based on the deletion of $P^{x}$-simple points, by applying a recent methodology that we proposed in [1. As it deletes solely $P^{x}$-simple points, this algorithm is guaranteed to preserve the topology. Furthermore, we have proposed some various sets $P$ such that our final algorithm deletes at least all the points removed by PK, while preserving the same end points. This also implies that PK is guaranteed to preserve the topology. In addition, our final algorithm also deletes points removed by the Gong and Bertrand's algorithm [16] (in the variant proposed by Rolland et al. [17]) while preserving the same end points.

In another study [132], we succeeded in proposing a new 12-subiteration thinning algorithm for 3D binary images, which produces curve or surface skeletons, and such that it deletes at least the points removed by one other 12-subiteration thinning algorithm [18]. A future work will propose new fully parallel thinning algorithms for 2D and 3D binary images.

\section{References}

1. C. Lohou and G. Bertrand. A new 3D 12-subiteration thinning algorithm based on P-simple points. In 8th IWCIA 2001, volume 46 of ENTCS, pages 39-58, 2001.

2. K. Palágyi and A. Kuba. A 3D 6-subiteration thinning algorithm for extracting medial lines. Pattern Recognition Letters, 19:613-627, 1998.

3. C.M. Ma and M. Sonka. A fully parallel 3D thinning algorithm and its applications. Computer Vision and Image Understanding, 64(3):420-433, 1996.

4. D.G. Morgenthaler. Three-dimensional simple points: Serial erosion, parallel thinning, and skeletonization. Technical Report TR-1009, Computer Vision Laboratory, University of Maryland, 1981.

5. T.Y. Kong. A digital fundamental group. Computer and Graphics, 13(2):159-166, 1989.

6. T.Y. Kong and A. Rosenfeld. Digital topology: introduction and survey. Computer Vision, Graphics and Image Processing, 48:357-393, 1989.

7. P.K. Saha, B. Chanda, and D.D. Majumder. Principles and algorithms for $2 \mathrm{D}$ and 3D shrinking. Technical Report TR/KBCS/2/91, N.C.K.B.C.S. Library, Indian Statistical Institute, Calcutta, India, 1991.

8. R.W. Hall. Connectivity preserving parallel operators in $2 \mathrm{D}$ and $3 \mathrm{D}$ images. In Vision Geometry, volume 1832 of SPIE, pages 172-183, 1992.

9. T.Y. Kong. On the problem of determining whether a parallel reduction operator for $n$-dimensional binary images always preserves topology. In Vision Geometry II, volume 2060 of SPIE, pages 69-77, 1993.

10. G. Bertrand. Simple points, topological numbers and geodesic numbers in cubic grids. Pattern Recognition Letters, 15:1003-1011, 1994.

11. C.M. Ma. On topology preservation in 3D thinning. Computer Vision, Graphics, and Image Processing: Image Understanding, 59(3):328-339, 1994. 
12. T.Y. Kong. On topology preservation in 2-D and 3-D thinning. International Journal of Pattern Recognition and Artificial Intelligence, 9(5):813-844, 1995.

13. T.Y. Kong. Topology-preserving deletion of 1's from 2-, 3- and 4-dimensional binary images. In 7th DGCI, volume 1347 of LNCS, pages 3-18, 1997.

14. S. Fourey and R. Malgouyres. A concise characterization of 3D simple points. In 9th DGCI, volume 1953 of $L N C S$, pages 27-36, 2000.

15. Y.F. Tsao and K.S. Fu. A parallel thinning algorithm for 3D pictures. Computer Graphics and Image Processing, 17:315-331, 1981.

16. W. Gong and G. Bertrand. A simple parallel 3D thinning algorithm. In International Conference on Pattern Recognition, pages 188-190, 1990.

17. F. Rolland, J.-M. Chassery, and A. Montanvert. 3D medial surfaces and 3D skeletons. In Visual Form 1991, pages 443-450, 1991.

18. K. Palágyi and A. Kuba. A parallel 3D 12-subiteration thinning algorithm. Graphical Models and Image Processing, 61:199-221, 1999.

19. G. Bertrand and Z. Aktouf. A three-dimensional thinning algorithm using subfields. In Vision Geometry III, volume 2356 of SPIE, pages 113-124, 1994.

20. K. Palágyi and A. Kuba. A hybrid thinning algorithm for 3D medical images. Journal of Computing and Information Technology, CIT 6, pages 149-164, 1998.

21. C.M. Ma. A 3D fully parallel thinning algorithm for generating medial faces. Pattern Recognition Letters, 16:83-87, 1995.

22. A. Manzanera, T. M. Bernard, F. Prêteux, and B. Longuet. A unified mathematical framework for a compact and fully parallel $n$-D skeletonization procedure. In Vision Geometry VIII, volume 3811 of SPIE, pages 57-68, 1999.

23. G. Bertrand. On P-simple points. Compte Rendu de l'Académie des Sciences de Paris, t. 321(Série 1):1077-1084, 1995.

24. G. Malandain and G. Bertrand. Fast characterization of $3 \mathrm{D}$ simple points. In IEEE International Conference on Pattern Recognition, pages 232-235, 1992.

25. G. Bertrand. Sufficient conditions for 3D parallel thinning algorithms. In Vision Geometry IV, volume 2573 of SPIE, pages 52-60, 1995.

26. G. Bertrand and R. Malgouyres. Some topological properties of discrete surfaces. In 6 th DGCI, volume 1176 of $L N C S$, pages 325-336, 1996.

27. J. Serra. Image analysis and mathematical morphology. Academic Press, 1982.

28. P.P. Jonker. Morphological operations on 3D and 4D images: From shape primitive detection to skeletonization. In 9th DGCI, volume 1953 of LNCS, pages 371-391, 2000.

29. G. Bertrand. P-simple points: A solution for parallel thinning. In 5th DGCI, pages 233-242, 1995.

30. R. Malgouyres and S. Fourey. Strong surfaces, surface skeletons and image superimposition. In Vision Geometry VII, volume 3454 of SPIE, pages 16-27, 1998.

31. J. Burguet and R. Malgouyres. Strong thinning and polyhedrization of the surface of a voxel object. In 9th DGCI, volume 1953 of $L N C S$, pages 222-234, 2000.

32. C. Lohou and G. Bertrand. A 3D 12-subiteration thinning algorithm based on $P$-simple points. Submitted for publication.

33. R.E. Bryant. Graph-based algorithms for boolean function manipulation. IEEE Transactions on Computer, Vol. C-35(8):677-691, 1986.

34. K.S. Brace, R.L. Rudell, and R.E. Bryant. Efficient implementation of a bdd package. In 27th IEEE Design Automation Conference, pages 40-45, 1990.

35. L. Robert and G. Malandain. Fast binary image processing using binary decision diagrams. Computer Vision and Image Understanding, 72(1):1-9, 1998. 